\section{P004 SERVICE EVALUATION OF A PATIENT CENTRED PATHWAY TO SUPPORT SELF MANAGEMENT AND WEIGHT LOSS FOR PATIENTS WITH NON-ALCOHOLIC FATTY LIVER DISEASE}

Mary Mccallum*, Ashis Mukhopadhya, Pauline Dundas. NHS Grampian, Aberdeen, UK

10.1136/gutjnl-2021-BASL. 13

With increasing rates of obesity, non-alcoholic fatty liver disease (NAFLD) is now the most common cause of abnormal liver function tests (LFTs) in the UK. ${ }^{1}$ Lifestyle change is the mainstay of clinical management for NAFLD. ${ }^{2}$ Patients achieving $\geq 5-10 \%$ weight reduction show regression in fibrosis score and improvements in histological aspects of NAFLD. ${ }^{3}$ However, there is limited evidence of what works to support weight loss/improve self management in clinical settings.

Evidence highlights just providing patients with NAFLD with information and advice to change behaviour is an insufficient intervention. Readiness to change weight-related behaviours is often low and not associated with severity of liver disease.

The Lead Clinician and Hepatology Nurse Specialist led on development of a new NAFLD pathway. They established a multidisciplinary clinic with input General Practitioners, dietitians and psychologists. Eligible patients (Fib 4>1.45) were offered input from a Liver Nurse, Health Psychologist, dietitian and Consultant.

Over 6 months patients received a low intensity psychology intervention. Each participant was seen for an assessment (and 8 weekly follow-ups via email, telephone or video call). Psychologist reviewed patients at three and six months.

101 patients, (53\% Males and 47\% Females) agreed to participate in the health psychology intervention. Average BMI of patients assessed was $40.1(113.5 \mathrm{~kg})$. Baseline daily step count was 1635. Majority of patients presented with two or more Long Term Conditions alongside NAFLD. Most common being Type 2 Diabetes (44\%) and anxiety/depression (27\%). Average Fib 4 score was 1.4, KPa 14.4. HbA1c 58.8, Cholesterol 5.0, ALT 81.4, AST 58.4.

$91 \%$ of patients were followed up (9\% drop out). Average weight loss $5.5 \mathrm{~kg}$ (range $0.5 \mathrm{~kg}-23 \mathrm{~kg}$ ). $82 \%$ increased daily step count (1635 to 5958). Anxiety scores (HADs) decreased from (7.4 to 6) and Depression scores reduced 6.3 to 3.3

Due to Covid 19 restrictions we don't have repeat measures on medical markers. However self reported outcomes at 6 months are encouraging.

A learning point when we are able to fully resume normal service delivery is to try and increase the number of patients benefitting from this service. We see scope to access patients earlier in primary care thus reducing waiting times and freeing up secondary care medical staff for more complex cases.

Overall, it is considered that this new pathway and multidisciplinary clinic has been very worthwhile. There were noticeable improvements identified through the use of appropriate assessment tools and from positive qualitative patient/ clinician feedback.

\section{REFERENCES}

1. Dyson JK, Anstee QM, McPherson S. Frontline Gastroenterology 2014;5:211-218.

2. Scenario: Management | Management | Non-alcoholic fatty liver disease (NAFLD) | CKS | NICE.

3. Vilar-Gomez E, Martinez-Perez Y, Calzadilla-Bertot L. Weight loss through lifestyle modification significantly reduces features of nonalcoholic steatohepatitis. Gastroenterology 2015;149:367-378.
P005 EVALUATING THE NON-VIRAL LIVER DISEASE BURDEN IN PEOPLE LIVING WITH HIV AND ABNORMAL LIVER ENZYMES

${ }^{1}$ Dev Katarey*, ${ }^{2}$ Yishi Tan, ${ }^{2}$ Laura Vickers, ${ }^{2}$ Harriet Sharpe, ${ }^{2}$ Bethany Parnell, ${ }^{2}$ Matija Tausan, ${ }^{1} J o n a t h a n$ Potts, ${ }^{2}$ Yvonne Gilleece, ${ }^{2}$ Sumita Verma. 'Royal Free Hospital, UKi ${ }^{2}$ Royal Sussex County Hospital

\subsection{6/gutjnl-2021-BASL.14}

Background Liver disease in the absence of viral hepatitis coinfection in people living with HIV (PLWH) is likely due to alcohol excess, metabolic syndrome (MS), and hepatotoxic antiretrovirals (ARV). We assessed the prevalence of hepatic fibrosis and associated risk factors in HIV mono-infected individuals with abnormal liver tests.

Methods PLWH with persistently elevated alanine aminotransferase (ALT) for $>6$ months were prospectively assessed using transient elastography, AUDIT questionnaire, and screening for MS. Thresholds for clinically significant hepatic fibrosis (CSHF) and cirrhosis were $>7.1 \mathrm{kPa}$ and $>12.5 \mathrm{kPa}$ respectively, and hepatic steatosis (HS) by a controlled attenuation parameter $($ CAP) $>237 \mathrm{~dB} / \mathrm{m}$.

Results Of 274 recruited individuals the median age was $52 \mathrm{yrs}$ (IQR 45-59), 93\% men, median HIV duration 15yrs (IQR 10-20), and undetectable viral load in 96\%. Overall, HS was seen in $169(61.7 \%)$ and CSHF was seen in $54(19.7 \%)$, of whom 19 (35.2\%) had cirrhosis and 40 (74.1\%) had HS. Alcohol, MS and ARV were implicated in 24 (44.4\%), 31 (57.4\%), and $15(27.8 \%)$ of patients with CSHF, respectively. No risk factors were identified in $10(18.5 \%)$ with CSHF. On binary and multinomial logistic regression lower HDL cholesterol (HR 0.255, 95\% CI $0.103-0.629, \mathrm{P}=0.003$ ) and diabetes $(\mathrm{HR} 2.558,95 \% \quad \mathrm{CI} 1.175-5.571, \quad \mathrm{P}=0.018)$ were independent predictors of CSHF. Both FIB-4 and APRI performed poorly in identifying CSHF (AUROC 0.556 and 0.603 respectively; figure 1).

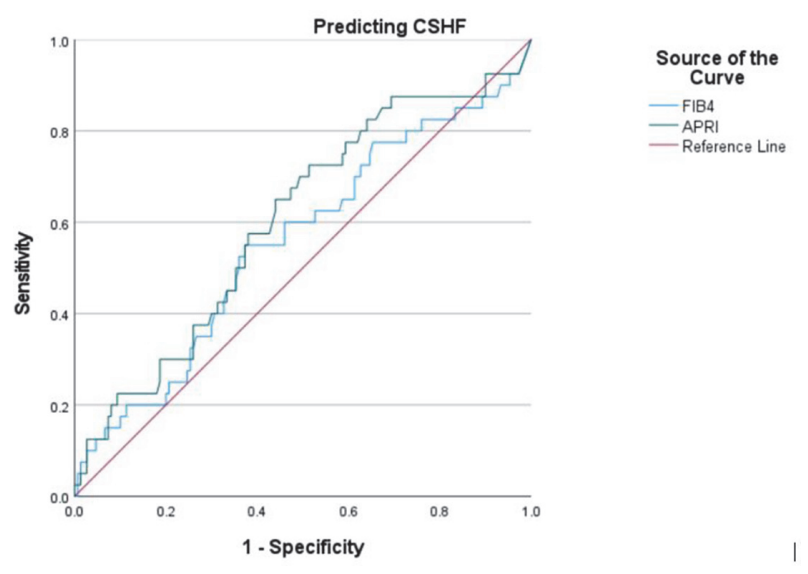

Abstract P005 Figure 1

Conclusion In these PLWH and elevated ALT, 20\% had CSHF and $\sim 60 \%$ had HS, in most cases associated with hazardous drinking or MS. Lower HDL and diabetes were independent predictor of CSHF. No risk factors were identified in almost 20\% raising the intriguing possibility that CSHF may be caused directly by the HIV infection. 